\title{
Infection during remission induction in childhood leukaemia
}

\author{
JUDITH M CHESSELLS AND ALISON D LEIPER
}

\author{
The Hospital for Sick Children, London
}

SUMMARY We have analysed our experience in the management of suspected infection in a group of 221 children with acute leukaemia undergoing induction of first remission. Patients with suspected infection received early empirical antibiotic therapy with cephalothin and gentamicin pending results of bacteriological investigations. Infection occurred in $17 \%$ of children with acute lymphoblastic leukaemia (ALL) whose initial treatment comprised prednisolone and vincristine, and was serious in $6.5 \% .27 \%$ of children with ALL treated with intensive induction had infections which were serious in $20 \%$; the figures for children with acute myeloblastic leukaemia (AML) were $49 \%$ and $22 \%$ respectively. The organisms responsible for most infections were Pseudomonas aeruginosa and Staphylococcus aureus; the former being most often associated with bacteraemia. One child $(0.5 \%)$ died from infection.

We conclude that with the use of early empirical antibiotic therapy, and granulocytes when appropriate, infection is no longer a major cause of death during remission induction. No special precautions are necessary to prevent its acquisition in most cases of ALL. In patients receiving early intensive treatment, including those with AML, measures designed to prevent acquisition of infection may reduce morbidity and enable the use of more effective chemotherapy.

Infection is the most common complication of remission induction in acute leukaemia. The incidence of infection during induction is proportional to the duration and severity of the neutropenia, almost inevitably present during the first 2 weeks of treatment in acute lymphoblastic leukaemia (ALL), and generally for several weeks in acute myeloblastic leukaemia (AML). There has been widespread acceptance of the concept of early antibiotic therapy in the febrile neutropenic patient, and of the value of granulocyte transfusions in the management of established infection; these reports usually concern large series of adult patients with AML. We have analysed our experience in management of infection in a group of children with acute leukaemia undergoing remission induction.

\section{Patients and methods}

The patients were children (aged up to 15 years) with acute leukaemia undergoing induction of first

Department of Haematology, The Hospital for Sick Children JUDITH M CHESSELLS, consultant clinical haematologist ALISON D LEIPER, leukaemia research fellow remission at The Hospital for Sick Children between September 1973 and January 1978. Most children were admitted to hospital for confirmation of the diagnosis and the first 2-3 days of treatment, but if well were discharged home as soon as possible thereafter, irrespective of their neutrophil counts. Children were generally admitted with a parent and were often nursed in a cubicle in which the mother also resided. Children with AML were always nursed in cubicles in simple reverse isolation. All patients received the normal ward diet and no use was made of skin decontamination or nonabsorbable antibiotics.

Antileukaemic treatment was started within 24-48 hours of diagnosis after treatment with allopurinol. Most children with ALL received prednisolone and vincristine with addition of L-asparaginase after recovery of their blood counts, usually during the third or fourth week of treatment. Some children with ALL with adverse prognostic features $\left(\mathrm{WBC}>20 \times 10^{9} / 1\right.$ before treatment, mediastinal mass, or aged more than 14 years) received more intensive initial treatment, usually with COAP (cyclophosphamide, cytosine arabinoside, vincristine, and prednisolone). Patients 
with AML received courses of TRAP (thioguanine daunorubicin, cytosine arabinoside, and prednisolone) or TRAV (substitution of vincristine for prednisolone). These two regimens were given as 5-day courses with 2- to 5-day rest periods between courses: most children with AML remained in hospital during the first two to four courses of drugs ( 3 to 6 weeks) but if well were allowed home during treatment-free periods between courses.

During this induction phase a standard protocol was followed for investigating and treating the child who had recurrent or sustained pyrexia $\left(>38^{\circ} \mathrm{C}\right)$. Cultures were taken of blood, nose, posterior pharyngeal wall, cough swab or sputum, rectum, urine, and any skin lesion present. A chest $x$-ray was performed and the cerebrospinal fluid was examined if there appeared to be a clinical indication.

Blood cultures were taken into Castenada's (aerobic) and Brewer's (anaerobic) medium and subcultured on day 7. Cough swabs, nose, and throat swabs were plated on to chocolate agar, blood agar, MacConkey's and Hoyle's medium. Mouth swabs and skin swabs were plated on to blood agar, MacConkeys' and Sabouraud's medium. Urine was plated on to CLED (cysteine lactose electrolyte deficient) medium, and faeces on to Salmonella Shigella agar and CLED medium.

After appropriate specimens were collected the pyrexial neutropenic child (neutrophils $<0.5 \times$ $\left.10^{9} / 1\right)$ was treated with IV antibiotics, gentamicin $8 \mathrm{mg} / \mathrm{kg}$ per day and cephalothin $80 \mathrm{mg} / \mathrm{kg}$ per day both given IV in four divided doses. Peak and trough levels of gentamicin were performed and the dose of gentamicin was adjusted accordingly. If there was a clinical suspicion of Pseudomonas aeruginosa infection (for example, skin or mucosal lesions) carbenicillin $20 \mathrm{~g} / \mathrm{m}^{2}$ per day, IV 4-hourly, was added to this regimen.

This empirical first-line combination was then adjusted according to the results of microbiological investigations. In proved bacterial infections treatment was continued for a minimum of 7 days and until the patient had been afebrile for at least 5 days. If infection could not be demonstrated the antibiotics were usually stopped after 5 days (see Results). Granulocyte transfusions were given, if available, to the febrile neutropenic child with proved serious infection in whom there was no immediate expectation of impending marrow recovery. From 1973 to 1975 granulocytes were obtained from donors with chronic myeloid leukaemia or from relatives by continuous flow centrifugation by Lowenthal et al. at the Hammersmith Hospital. ${ }^{1}$ From 1976 onwards granulocytes were obtained from relatives and ABO $\mathrm{Rh}$ compatible donors using a Haemonetics cell separator.
Investigation for possible viral infections was not performed routinely on all cases, only on children with persistent pyrexial illness unresponsive to antibiotics and apparently not attributable to bacterial infection.

We attempted to classify the results according to the criteria described by the antimicrobial therapy project group of the European Organisation for Research on Treatment of Cancer. ${ }^{2}$ This classification is shown in Table 1 . We also attempted to define the incidence of serious infections; this was necessarily a subjective exercise but in general serious infections comprised all bacteraemias and severe pneumonia or cellulitis.

\section{Results}

The results of investigations and the outcome of treatment in the whole group of 221 children are shown in Table 2 . Although $40 \%$ of children with ALL had an episode of pyrexia during remission induction, infection was confirmed or probable in less than one-half of these and serious infection occurred in less than one-quarter. In contrast probable infection occurred in one-half of all children with AML and serious infection in almost one-quarter. Episodes of pyrexia were common and only 3 children with AML completed 8 weeks' treatment without a proved or suspected infection.

Microbiologically confirmed infections (class 1). In Table 3 are listed the organisms isolated in the patients with microbiologically proved infections.

\section{Table 1 Classification of infections (EORTC, 1978) ${ }^{2}$}

I Microbiologically confirmed infection with bacteraemia

without bacteraemia

II Clinically confirmed infections: signs and symptoms with an identifiable site but without microbiological proof of the aetiological agent

III Probable infection: equivocal signs and symptoms of infection with no microbiological confirmation

IV Doubtful infection: in retrospect the febrile episode was probably not due to infection

Table 2 Incidence of infection

\begin{tabular}{|c|c|c|c|c|}
\hline & \multicolumn{2}{|c|}{$A L L(n=184)$} & \multicolumn{2}{|c|}{$A M L(n=37)$} \\
\hline & No. & $\%$ & No. & $\%$ \\
\hline $\begin{array}{l}\text { Pyrexial during induction } \\
\text { Confirmed or probable }\end{array}$ & 73 & 40 & 31 & 84 \\
\hline $\begin{array}{l}\text { infection } \\
\text { Doubtful infection }\end{array}$ & $\begin{array}{l}34 \\
39\end{array}$ & $\begin{array}{l}18 \\
22\end{array}$ & $\begin{array}{l}18 \\
13\end{array}$ & $\begin{array}{l}49 \\
35\end{array}$ \\
\hline $\begin{array}{l}\text { Serious infections } \\
\text { Death during first } 8 \text { weeks of }\end{array}$ & 16 & 9 & 8 & 22 \\
\hline $\begin{array}{l}\text { treatment } \\
\text { Death primarily due to } \\
\text { infection }\end{array}$ & $\begin{array}{l}3 \\
1\end{array}$ & $1 \cdot 6$ & 5 & 14 \\
\hline
\end{tabular}


Table 3 Microbiologically confirmed infections

\begin{tabular}{|c|c|c|}
\hline & $A L L$ & $A M L$ \\
\hline $\begin{array}{l}\text { Bacteraemia } \\
\text { Pseudomonas aeruginosa } \\
\text { Escherichia coli } \\
\text { Staphylococcus aureus } \\
\text { Streptococcus pneumoniae } \\
\text { B-Haemolytic streptococcus } \\
\text { Klebsiella pneumoniae }\end{array}$ & $\begin{array}{l}6 \\
4 \\
1 \\
1 \\
- \\
-\end{array}$ & $\begin{array}{l}1 \\
\frac{1}{-} \\
1 \\
1\end{array}$ \\
\hline $\begin{array}{l}\text { No bacteraemia } \\
\text { Pseudomonas aeruginosa } \\
\text { Staphylococcus aureus } \\
\text { B-Haemolytic streptococcus } \\
\text { Escherichia coli } \\
\text { Mycoplasma pneumoniae } \\
\text { Candida albicans }\end{array}$ & $\begin{array}{l}1(1) \\
8(4) \\
2 \\
1 \\
-1\end{array}$ & $\begin{array}{l}4(4) \\
3(1) \\
4 \\
-2 \\
4(2)\end{array}$ \\
\hline
\end{tabular}

Figures in brackets correspond to serious nonbacteraemic infections Numbers do not correspond to those in Table 2 as some children had multiple infections.

The organisms responsible for most infections were $P$. aeruginosa and Staphylococcus aureus. Pseudomonas was responsible for the largest number of bacteraemic infections ( 7 patients, 6 of whom had associated cellulitis or rectal ulceration). All these children were treated with carbenicillin and gentamicin except one in whom the organism was resistant to carbenicillin who received tobramycin. Granulocyte transfusions were given to 6 of the 7 bacteraemic patients; the 7th, a boy with AML in his fourth treatment week, recovered with antibiotics and achieved remission one week later. One girl with ALL died despite treatment with antibiotics and granulocytes.

$S$. aureus was responsible for one episode of bacteraemia in a girl with ALL and skin lesions, 3 cases of pneumonia (one with meningitis) in children with ALL, 2 serious cases of cellulitis (one in ALL), and 6 less serious skin infections. One of the 3 children with pneumonia died of renal failure and pulmonary haemorrhage; the other children all recovered. Granulocytes were given to the patients with bacteraemia with meningitis and to one girl with AML and cellulitis.

Five children had Escherichia coli bacteraemia, one associated with pneumonia and one with skin sepsis, and a 6th child had a urinary tract infection. All 5 septicaemic children recovered although one developed subsequent pseudomonas infection and died. Granulocytes were given to 4 of the 5 bacteraemic children.

The single episode of pneumococcal bacteraemia took place in a child with pneumonia, the group A $\beta$-haemolytic streptococcal infection was associated with cellulitis, and the child with bacteraemia due to Klebsiella pneumoniae had AML resistant to remission induction but no localised signs of infection. Candidiasis occurred in 5 children: in one with ALL and 4 with AML. Slight infection in 3 children responded to oral nystatin. Severe mucocutaneous infection occurred in 2 children both with AML, but responded to oral and topical nystatin and amphotericin. Candida septicaemia was not observed.

Clinically diagnosed infections (class II). These occurred in 9 children with ALL and one was deemed serious: a boy with persistent abdominal pain and pyrexia, treated with gentamicin cephalothin, and carbenicillin, eventually developed signs of a subphrenic abscess necessitating laparotomy and removal of a spleen with multiple abscesses. Four patients had acute otitis media, 3 clinical and radiological signs of pneumonia, and one diarrhoea and vomiting for which no infective cause was found although other family members had similar symptoms.

Four children with AML developed otitis media during remission induction and 2 had clinical signs and minor $x$-ray changes of pneumonia.

Probable infection (class III). Two children with ALL were thought to have clinical evidence of infection; both had severe neutropenia and persistent swinging pyrexia but in neither did bacteriological or virological cultures show a source of infection. Recovery of the neutrophil count was associated with resolution of the fever in both of them.

Doubtful infection (class IV). Many children with ALL (39) had episodes for which no infective cause was found. In 33 the fever was present during the first week of treatment and settled within one to 3 days of the start of treatment with antibiotics. One or more episodes of fever without apparent infective cause took place in 13 of the children with AML. These were seen with no particular frequency during any one week of treatment.

\section{Mortality}

The number of children dying within the first 8 weeks of start of treatment is shown in Table 2. Only one death was primarily due to infection: this was a child with ALL who developed E. coli septicaemia and subsequently pseudomonas, cellulitis, and septicaemia. One child with ALL died from renal failure, and pulmonary and pericardial haemorrhage within 6 days, and one child with intracranial haemorrhage died 48 hours after admission. Three deaths in children with AML, all due to intracranial haemorrhage, occurred within 24 hours of admission; one child died from progressive disease at 5 weeks and one from renal failure at 2 weeks. 


\section{Morbidity}

Four children with ALL experienced long-term sequelae as a result of their initial infections. Two required skin grafting because of skin necrosis due to pseudomonas cellulitis. One of these, a boy whose stormy induction course necessitated 20 granulocyte transfusions for the management of his infection, later developed chronic cytomegalovirus infection and died of cor pulmonale 4 years after his initial diagnosis. We are not sure whether or not the infection resulted from his repeated exposure to blood products. One girl with staphylococcal pneumonia and meningitis developed a bronchopulmonary fistula which healed spontaneously and she now remains well off treatment 5 years later. One boy who had splenectomy for multiple abscesses one year later developed postsplenectomy septicaemia due to Haemophilus influenzae while on prophylactic penicillin, and died of fulminating infection.

Relationship of induction regimen to acquisition of infection. The prevalence of infection in ALL according to induction regimen is illustrated in Table 4. The incidence of probable, proved, and serious infection is the same for children with 'standard risk' or 'poor risk' ALL when treated with prednisolone and vincristine. The use of more intensive initial treatment is associated with a higher incidence of probable and serious infection, but not with a higher death rate. The only death from infection in ALL was in a girl in the standard risk group who received prednisolone and vincristine only.

Time of acquisition of infections (Table 5). We looked at the time from the start of treatment when the more common microbiologically proved infections

Table 4 Infection according to induction regimen in $A L L$

\begin{tabular}{lllllllll}
\hline & Pyrexial & Infection & $\begin{array}{c}\text { Serious } \\
\text { infection }\end{array}$ & Death \\
\cline { 2 - 6 } & No. & $\%$ & No. & $\%$ & No. & $\%$ & \\
\hline $\begin{array}{c}\text { 'Standard' risk patients } \\
\text { (n = 117) }\end{array}$ & 43 & 37 & 20 & 17 & 7 & 6 & 1 \\
$\begin{array}{c}\text { Poor' risk patients } \\
\text { Standard treatment } \\
(\mathrm{n}=37)\end{array}$ & 15 & 40 & 6 & 16 & 3 & 8 & 1 \\
$\begin{array}{c}\text { Intensive treatment } \\
(\mathrm{n}=30)\end{array}$ & 15 & 50 & 8 & 27 & 6 & 20 & 1 \\
\hline
\end{tabular}

'Poor' risk patient, mediastinal mass, WBC $>20 \times 109 / 1$ before treatment, or aged more than 14. 'Standard' risk, none of these features. Standard treatment, prednisolone and vincristine initially. Intensive treatment, additional drugs (see text).
Table 5 Time of onset of infections

\begin{tabular}{|c|c|c|c|c|c|c|c|c|}
\hline \multirow[t]{2}{*}{ Organism } & \multicolumn{8}{|c|}{ Week of treatment } \\
\hline & 1 & 2 & 3 & 4 & 5 & 6 & 7 & 8 \\
\hline $\begin{array}{c}\text { Pseudomonas } \\
\text { aeruginosa }\end{array}$ & $3(1)$ & 2 & $3(2)$ & $1(1)$ & $2(1)$ & - & 1 & 一 \\
\hline Escherichia coli & 3 & $3(1)$ & - & - & - & - & - & - \\
\hline $\begin{array}{l}\text { Staphylococcus } \\
\text { aureus } \\
\beta \text {-Haemolytic }\end{array}$ & $5(1)$ & 3 & $3(1)$ & - & 一 & - & - & $1(1)$ \\
\hline $\begin{array}{l}\text { streptococcus } \\
\text { Candida }\end{array}$ & s $3(1)$ & 一 & $2(2)$ & 一 & $1(1)$ & 一 & 一 & $1(1)$ \\
\hline albicans & $2(2)$ & $2(1)$ & - & - & - & - & $1(1)$ & - \\
\hline Total & $16(5)$ & 10 (2) & $8(5)$ & $1(1)$ & 3 (2) & - & $2(1)$ & $2(2)$ \\
\hline
\end{tabular}

Figures refer to total number of infections due to each organism. Numbers in brackets are children with AML.

occurred. As expected those in patients with ALL were usually seen during the first two weeks of treatment; the two late pseudomonas infections at 5 and 7 weeks were in children with ALL who were slow to remit and who had remained in hospital. No child with ALL who was discharged home was subsequently readmitted with confirmed or probable infection.

\section{Discussion}

Serious bacterial infection occurred in $6.5 \%$ of children with ALL whose initial treatment comprised prednisolone and vincristine. Thus infection, although rare, may cause morbidity or death in children who might otherwise experience long-term disease-free survival.

The incidence of infection in our patients is close to that reported by others. The only similar group of children reported from a single centre had an identical incidence of serious infection. ${ }^{3}$ In the multicentre UKALL I trial $9 \%$ of 198 children had bacteraemia or serious pneumonia during induction with prednisolone and vincristine, and there were two deaths from infection during induction. ${ }^{4}$

We have also shown, not unexpectedly, that intensification of early treatment in ALL carries the risk of a higher incidence of infection, and the implications of this are discussed below.

A greater number of children with AML were pyrexial and had proved infections although no child died as a result of infection during the induction period. An analysis of early death in patients with AML suggests that death is most often due to bacteraemia associated with local infection, and occurs in patients whose disease is slow to respond to chemotherapy. ${ }^{5}$ Our results suggest that younger patients have a greater ability to withstand marrow hypoplasia induced by treatment and confirms a similar finding by other workers. ${ }^{6}$ Our 3 early deaths 
from intracranial haemorrhage might possibly have been avoided by a more prompt and liberal use of platelet concentrates immediately after admission.

What lessons have we learned from our experience in management of the febrile neutropenic child? Our policy of early empirical treatment with antibiotics gave satisfactory results and was associated with a low death rate. In retrospect many patients received antibiotics unnecessarily. Could this overuse of antibiotics have been avoided? Most of the febrile episodes in children with ALL which were classified as 'doubtful infection' took place during the first week of treatment and settled within 2-3 days. However there was no sure way of determining at the onset of the episode whether or not the child would prove to have bacteraemia. The febrile episodes in children with AML were not clustered in any particular week of treatment. Most patients in whom bacteraemia was proved also had skin lesions or changes in their chest $x$-rays, but this was not true for all patients, particularly those with infections due to Gram-negative organisms. In view of the rapidity with which deterioration and death can occur in Gram-negative bacteraemia early broad spectrum antibiotic treatment must be given to the febrile neutropenic patient. ${ }^{7}$

Our choice of antibiotics was modified once the result of cultures had become available but it seems that our initial choice although effective was not the best one. We did not see nephrotoxicity due to the combination of cephalothin and gentamicin in this group of patients but this complication has been reported. ${ }^{8}$ We have ourselves seen nephrotoxicity in children with aplastic anaemia who received this antibiotic combination during repeated pyrexial episodes.

$P$. aeruginosa was the organism most often responsible for bacteraemia in our patients and therefore the combination of gentamicin and carbenecillin is a more logical first choice and the one which we have now adopted. This combination has recently been demonstrated by the EORTC antimicrobial project group to be superior to others containing cephalothin (cephalothin and carbenecillin, cephalothin and gentamicin) as initial empirical treatment in granulocytopenic patients. ${ }^{2}$ The group suggest that if there is no response to antibiotics these should be stopped once the possibility of bacterial infection can reasonably be discounted-after about 96 hours. This'policy should reduce toxicity and the unnecessary use of antibiotics. It is important in patients apparently responding to antibiotics to continue treatment for a minimum of 7-10 days even if cultures prove sterile; too early cessation of treatment may cause relapse of infection and death. ${ }^{9}$
We may have failed to diagnose viral infections in the group of children with unexplained pyrexia. The indications for virological investigation were restricted but it did not seem as if viral infections were a major cause of morbidity in this group of patients. Nonbacterial infections assume greater importance in patients who are immunosuppressed after prolonged chemotherapy.

It is difficult to assess the benefit of granulocyte transfusions in our patients as they were not given as a controlled trial and during the earlier years they were not always available. Most of our patients with Gram-negative bacteraemia received granulocytes and it is in such patients that these appear to be of most benefit. ${ }^{10}$ In ALL treated with prednisolone and vincristine, when there is expectation of early marrow recovery, granulocyte transfusions are only likely to be indicated in a few patients-such as those in our series with early pseudomonas of $E$. coli infection.

Should we adopt a more rigorous approach to the prevention of infection? There appears from our results to be no indication for such an approach during standard induction of ALL, and outpatient treatment or early discharge home is clearly in the best interests of most patients and their families. No patient with ALL in this series discharged from hospital was readmitted with serious infection and therefore there seems no justification for a prolonged stay in hospital.

These children were all undergoing first remission induction. Greater infective problems might be anticipated in children undergoing reinduction after relapse during chemotherapy. In theory there might be a case for more stringent measures to prevent infection in such children with resistant ALL. However we have recently used a combination of prednisolone, vincristine, daunorubicin, and Lasparaginase in children relapsing during treatment and have adopted the criteria here described for admission to hospital and for treatment of suspected infection. Remission was achieved in over $90 \%$ of patients without any deaths from infection. ${ }^{18}$ Children relapsing during treatment have an extremely poor prognosis and prolonged stay in hospital would seem to be even less desirable for them than for other patients. ${ }^{11}$

What attitude should be adopted in AML? Our patients were isolated in an attempt to prevent acquisition of infection while in hospital but were allowed home during treatment-free periods in hospital. Only $3(8 \%)$ of our patients with AML escaped a course of antibiotics although no patient died with infection. The rate of acquisition of infection during treatment can be reduced by reverse isolation, oral nonabsorbable antibiotics, and 
topical antiseptics. ${ }^{12}$ It remains to be seen whether such measures by enabling more intensive treatment will influence remission rate or survival, but in one recent report this seemed to be the case. ${ }^{13}$ The combination of trimethoprin-sulphamethoxazole, of proved benefit in prevention of pneumocystis infection in children with ALL, ${ }^{14}$ has recently been used to prevent or delay infection in neutropenic patients with acute leukaemia. ${ }^{15}$ However the use of co-trimoxazole as a preventive agent or as first-line treatment will not obviate the risk of infection with pseudomonas and may increase the incidence of resistant organisms. ${ }^{16} \mathrm{~A}$ controlled trial of prophylactic co-trimoxazole in neutropenic patients is at present being conducted by the EORTC. If this drug proves helpful in preventing infection, its use should certainly be considered in children with AML.

Our results in patients with ALL show that children with low and high leucocyte counts at presentation have an equal risk of infection but that this risk is increased by intensive early treatment with combinations such as COAP. Similarly early treatment with L-asparaginase in the UKALL $I$ trial was associated with more serious infections and deaths than treatment with prednisolone and vincristine alone as in UKALL I. ${ }^{4}$ However the infections in our patients all responded to treatment with antibiotics and granulocytes where appropriate.

It is possible that treatment with multiple cytotoxic agents early during induction may achieve an increased duration of subsequent remission. An analysis of factors influencing duration of haematological remission in ALL has suggested that consolidation therapy or use of drugs additional to prednisolone and vincristine may be beneficial. ${ }^{17}$ There has been no improvement in the results of treatment of ALL for the last 5 years, and few patients except perhaps girls with low leucocyte counts, can truly be deemed 'good risks'. In present circumstances a trial of early intensification of treatment seems clearly justified. The price of such treatment is increased risk of infection, but with adequate supportive facilities it should be possible for such complications to be diagnosed early and treated promptly.

We thank the members of the department of microbiology, particularly Dr W C Marshall, The Hospital for Sick Children, for help, and Dr Beryl Jameson, Royal Marsden Hospital for encouragement.

ADL is supported by the Leukaemia Research Fund.

\section{References}

1 Lowenthal R M, Grossman L, Goldman J M, et al. Granulocyte transfusions in treatment of infections in patients with acute leukaemia and aplastic anaemia. Lancet 1975 ; 1 : 353-8.

2 European Organisation for Research on Treatment of Cancer: International Antimicrobial Project Group. Three antibiotic regimens in the treatment of infection in febrile granulocytopenic patients with cancer. $J$ Infect Dis 1978; 137: 14-29.

3 Hughes W T, Smith D R. Infection during induction of remission in acute lymphocytic leukemia. Cancer 1972; 31: 1008-14.

4 Johnston P G B, Hardisty R M, Kay H E M, Smith P G. Myelosuppressive effect of colaspase (L-asparaginase) in the initial treatment of acute lymphoblastic leukaemia. Br Med J 1974; iii: 81-3.

5 Smith I E, Powles R, Clink H McD, Jameson B, Kay H E M, McElwain T J. Early deaths in acute myelogenous leukemia. Cancer 1977; 39: 1710-4.

- Medical Research Council Working Party on Leukaemia in Adults. Treatment of acute myeloid leukaemia with daunorubicin, cytosine arabinoside, mercaptopurine, L-asparaginase, prednisone, and thioguanine: results of treatment with five multiple drug schedules. Br J Haematol 1974; 27 : 373-89.

7 Gaya $H$. The treatment of infection in acute leukaemia. Br J Hosp Med 1975; 13: 124-9.

8 Fillastre J P, Laumonier R, Humbert G, et al. Acute renal failure associated with combined gentamicin and cephalothin therapy. $B r$ Med $J$ 1973; ii: 396-7.

2 Rodriguez V, Burgess M, Bodey G. Management of fever of unknown origin in patients with neoplasms and neutropenia. Cancer 1973; 32: 1007-12.

10 Graw R G, Jr, Herzig G, Perry S, Henderson E S. Normal granulocyte transfusion therapy. $N$ Engl J Med 1972; 287: 367-72.

11 Cornbleet M A, Chessells J M. Bone-marrow relapse in acute lymphoblastic leukaemia in childhood. $\mathrm{Br} \mathrm{Med} \mathrm{J}$ 1978; ii: $104-6$.

12 Storing R A, Jameson B, McElwain T J, Wiltshaw E, Spiers A S D, Gaya H. Oral non-absorbed antibiotics prevent infection in acute non-lymphoblastic leukaemia. Lancet 1977; 2: 837-40.

13 Rodriguez V, Bodey G P, Freireich E J, et al. Randomized trial of protected environment-prophylactic antibiotics in 145 adults with acute leukemia. Medicine (Baltimore) $1978 ; 57: 253-66$.

14 Hughes W T, Kuhn S, Chaudhary S, et al. Successful chemoprophylaxis for Pneumocystis carinii pneumonitis. $N$ Engl J Med 1977; 297: 1419-26.

15 Enno A, Catovsky D, Darrell J, Goldman J M, Hows J, Galton D A G. Co-trimoxazole for prevention of infection in acute leukaemia. Lancet 1978; 2: 395-7.

16 Editorial: Infection prevention in acute leukaemia. Lancet 1978; 2: 769-70.

17 Simone J V. Factors that influence haematological remission duration in acute lymphocytic leukaemia. Br J Haematol 1976; 32: 465-72.

18 Chessells J M, Cornbleet M. Combination chemotherapy for bone marrow relapse in childhood lymphoblastic leukemia (ALL). Med Pediatr Oncol 1979; 6: 359-65.

Correspondence to Dr J M Chessells, Department of Haematology, The Hospital for Sick Children, Great Ormond Street, London WCIN 3JH.

Received 2 March 1979 